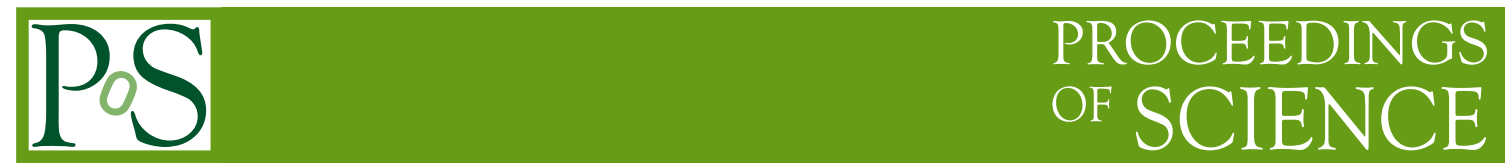

\title{
Calibration and Imaging with eigenbeams
}

\author{
Maxim Voronkov* and Tim Cornwell \\ CSIRO Australia Telescope National Facility \\ E-mail: Maxim.Voronkov@csiro.au, Tim.Cornwell@csiro.au
}

\begin{abstract}
The Australian Square Kilometre Array Pathfinder (ASKAP) is one of the key technology demonstrators on the way to the Square Kilometre Array (SKA). The main feature of the project is the development of the Phased Array Feed (PAF) technology to boost the instantaneous field of view. We consider a non-adaptive (weights are constant) beamforming and show that the majority of information is contained in a small number of linear combinations of the measured voltages. Therefore, a small number of synthetic beams, perhaps an order of magnitude smaller than the number of physical feeds, can be used for imaging and calibration. From the other side, only a small number of gains can be calibrated using the full-beam self-calibration approach.
\end{abstract}

From Planets to Dark Energy: the Modern Radio Universe

October 1-5 2007

The University of Manchester, UK

${ }^{*}$ Speaker. 


\section{Introduction}

Wide instantaneous field of view is one of the key science requirements for the Square Kilometre Array (SKA) as it directly affects the survey speed. A significant experience necessary to overcome the challenges of the wide field of view regime can be gained designing the SKA technology demonstrators, such as the Australian Square Kilometre Array Pathfinder (ASKAP). This radio-interferometer will have an array of feeds mounted in the focal plane of each dish in order to achieve a better performance than conventional interferometers can provide (wider instantaneous field of view and better illumination of the aperture). The elements of each feed array are phased together in the beamformer (hence, the term Phased Array Feed or PAF). It reduces the amount of correlated information by forming a certain number of the linear combinations from the signals received by each feed. Although the number of correlator inputs per antenna is less than the number of feeds, a freedom to choose weights of the linear combinations allow different optimization strategies. In this paper we consider a non-adaptive (weights are constant) beamforming from the imaging and calibration perspective. A more detailed overview of this work can be found in [1].

\section{Eigenbeams and optimization}

The beamformer calculates a linear combination of inputs giving a synthetic voltage pattern

$$
E(\vec{s})=\sum_{l} w_{l} E_{l}(\vec{s})
$$

where $E_{l}(\vec{s})$ is a voltage pattern of the $l$ th element and $w_{l}$ is a corresponding complex weight. The power beam is a quadratic form, which can be optimized using methods of linear algebra

$$
A(\vec{w}, \vec{s})=E(\vec{s}) E^{*}(\vec{s})=\sum_{l, m} w_{l}^{*} E_{l}^{*} w_{m} E_{m}=\vec{w}^{H} \mathscr{E}(\vec{s}) \vec{w},
$$

where $\mathscr{E}(\vec{s})$ is a voltage pattern matrix $\mathscr{E}(\vec{s})=\left\|E_{l}^{*}(\vec{s}) E_{m}(\vec{s})\right\|_{m}^{l}$ for direction $\vec{s}$. One can maximize

$$
F(\vec{w})=\int A(\vec{w}, \vec{s}) K(\vec{s}) d \vec{s}=\vec{w}^{H}\left[\int \mathscr{E}(\vec{s}) K(\vec{s}) d \vec{s}\right] \vec{w}=\vec{w}^{H} \mathscr{E} \vec{w}
$$

where the matrix $\mathscr{E}=[\cdots]$ is direction independent, and $K(\vec{s})$ is an arbitrary kernel, which essentially defines the desired optimization. This quadratic form attains its maximum under condition of $\vec{w}^{H} \vec{w}=1$, if $\vec{w}$ is an eigenvector of $\mathscr{E}$ corresponding to the largest eigenvalue. If the beamformer has multiple outputs (i.e. the observations can be performed with a number of different weight vectors simultaneously), eigenvectors corresponding to the appropriate number of the largest eigenvalues can be used. The optimization with $K(\vec{s})=1$ and $\mathscr{E}$ corresponding to the actual sky has a physical meaning of maximizing the flux from celestial sources collected in the field of view. This approach can be used for calibration. Fig. 2 illustrates the first eigenbeam and eigenspectrum generated for some NVSS field. The eigenvalues decrease in magnitude very steeply, which means that a small number of eigenbeams contains the majority of information. From another point of view, the amount of information may not be enough to constrain the calibration solution for a large number of element gains by observing the same region on the sky (fixed configuration of sources). Using a 

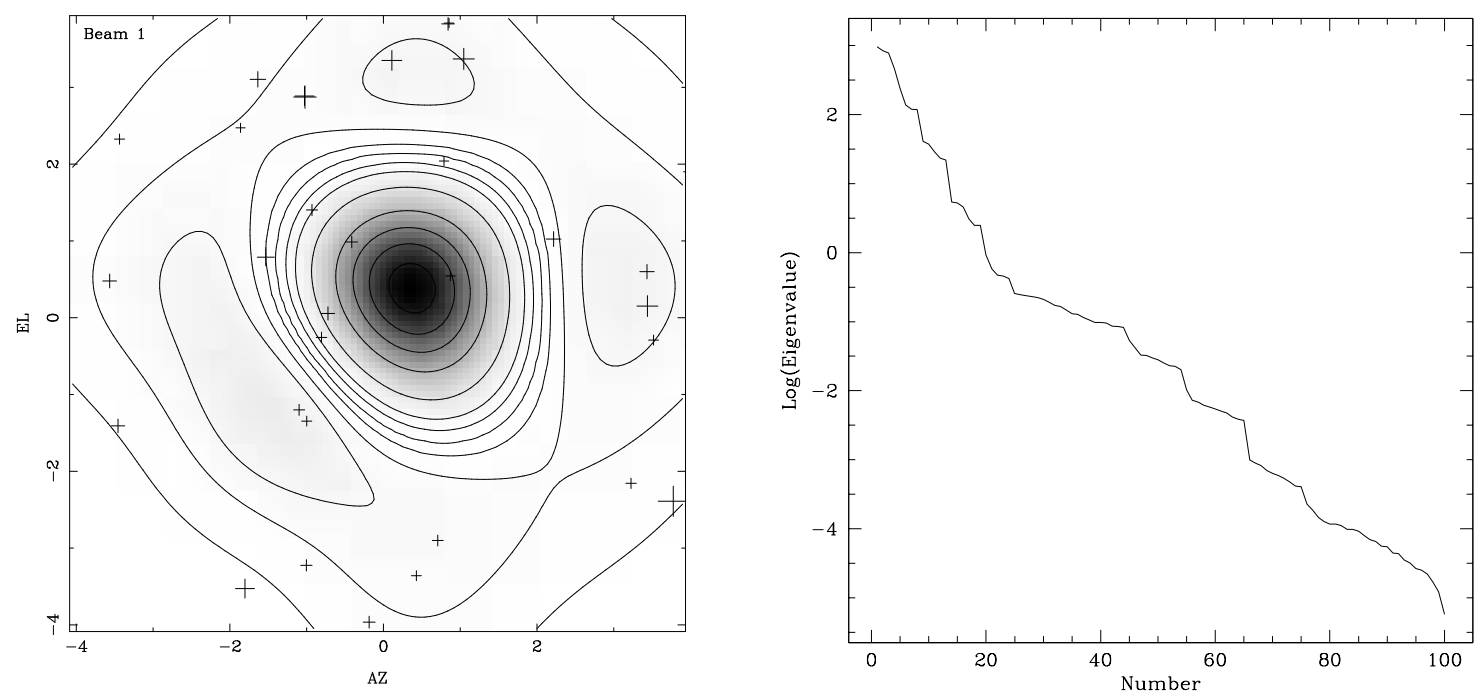

Figure 1: Beams corresponding to the first eigenvector obtained using an NVSS field as a sky model (left) and the corresponding eigenvalue spectrum in the logarithmic scale (right). Crosses on the left-hand image mark the positions of sources (the cross size represent the flux).

uniform mesh of sources as $\mathscr{E}$ and a tapering Gaussian as $K(\vec{s})$, allows to produce beams with more regular sidelobe properties (Fig. 2). Such beams can be used for imaging, which appear to be quite similar to an ordinary linear mosaicing. It is worth noting, that the current analysis does not take into account the spill-over and the noise properties of eigenbeams. A generalization can be made by analogy to [2], although the appropriate simulations would require a more elaborated model of the PAF installation mounted on the dish.

\section{Conclusions}

1. A relatively small number of synthetic beams (linear combinations in the beamformer) contains most of the information.

2. Only a small number of linear combinations of gains can be determined in the full-beam selfcalibration procedure (using all known sources in the field of view). Therefore, one needs to track the relative gains somehow (e.g. by a noise source in the dish vertex illuminating the whole focal plane array installation).

\section{References}

[1] M.A. Voronkov, T.J. Cornwell, On the calibration and imaging with eigenbeams, ATNF SKA Memo 12, [ http://www.atnf.csiro.au/SKA/newdocs/eigenbeams.pdf ]

[2] Brisken W., Craeye C., 2004, Focal Plane Array Beam-forming and Spill-over Cancellation using Vivaldi Antennas, EVLA Memo 69;

[http://www.aoc.nrao.edu/evla/geninfo/memoseries/evlamemo69.pdf] 

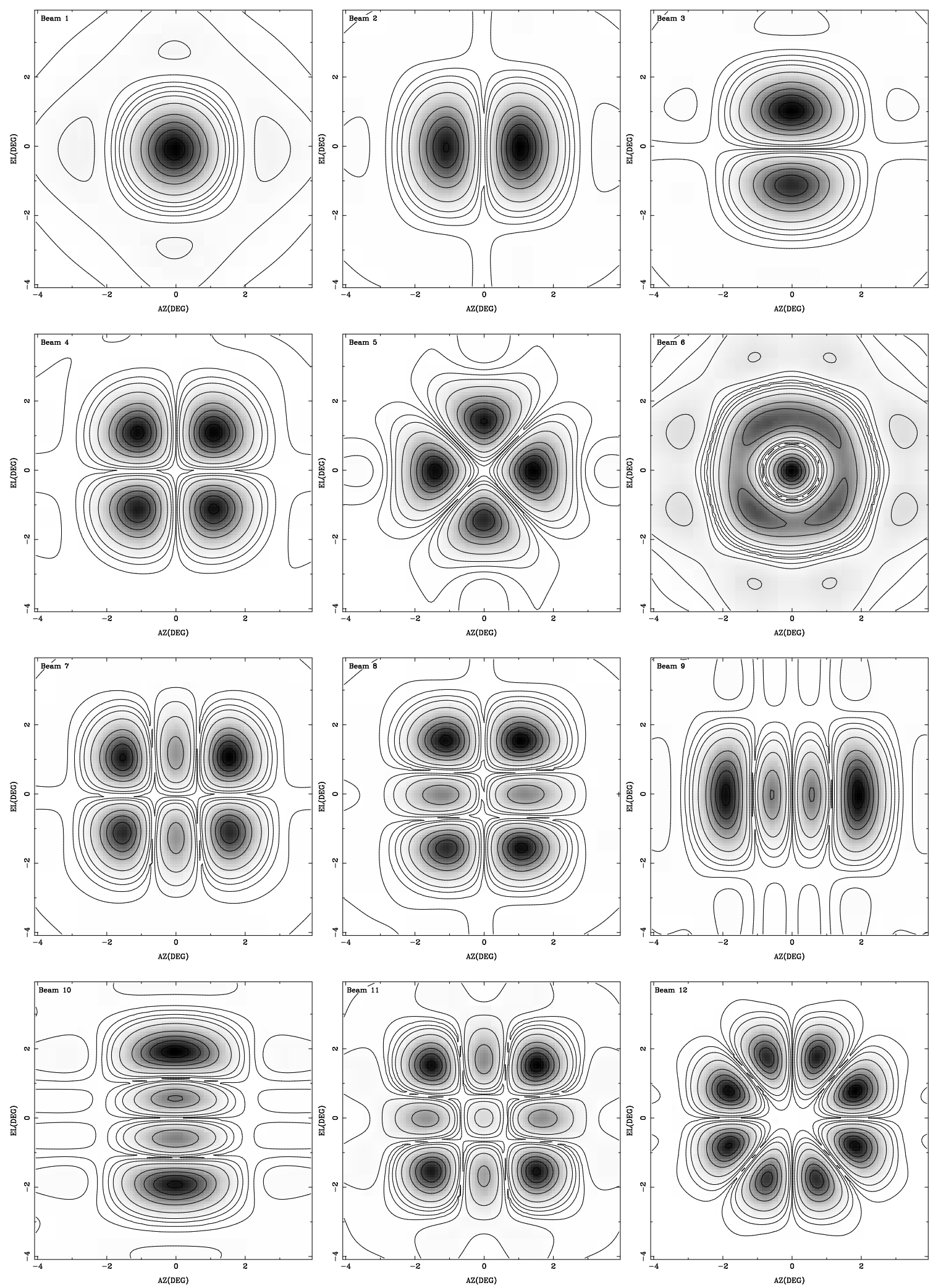

Figure 2: Eigenbeams corresponding to 12 largest eigenvalues. The simulations assumed observations at $1.4 \mathrm{GHz}$ with a $15 \mathrm{~m}$ dish and a $10 \times 10$ array of feeds with a 20 arcmin separation. Contours are at $0.1,1,3$, $10,30,50,70$ and $90 \%$. 